\title{
Effect of Permeability on Implicit-Texture Foam Model Parameters and the Limiting Capillary Pressure
}

\author{
R. Farajzadeh, ${ }^{* \dagger, \dagger}$ M. Lotfollahi, ${ }^{\S}$ A. A. Eftekhari, ${ }^{\dagger}$ W. R. Rossen, ${ }^{\dagger}$ and G. J. H. Hirasaki ${ }^{\prime \prime}$ \\ †Delft University of Technology, Stevinweg 1, 2628 CN Delft, The Netherlands \\ ${ }^{\ddagger}$ Shell Global Solutions International, 2280 AB Rijswijk, The Netherlands \\ ${ }^{\S}$ University of Texas at Austin, Austin, Texas 78712, United States \\ "Rice University, Houston, Texas 77251, United States
}

Supporting Information

ABSTRACT: Accurate modeling of foam rheology on the field scale requires detailed understanding of the correlation between the fundamental properties of foam and the scalable parameters of the porous medium. It has been experimentally observed that foam experiences an abrupt coalescence when the capillary pressure in the porous medium approaches a certain value referred to as the "limiting capillary pressure", $P_{\mathrm{c}}{ }^{*}$. Current foam models that treat foam texture implicitly mimic this fundamental behavior with a so-called dry-out function, which contains adjustable parameters like fmdry and epdry (in the STARS foam simulator). Parameter fmdry (called $S_{\mathrm{w}}{ }^{*}$ in other models) represents the water saturation corresponding to the limiting capillary pressure, $P_{c}{ }_{c}^{*}$, and epdry determines the abruptness of foam coalescence as a function of water saturation. In this paper, using experimental data, we examine the permeability dependence of these parameters. We find that the value of fmdry decreases with increasing permeability. We also find that, for the data examined in this paper, the transition from the high-quality regime to low-quality regime is more abrupt in lower-permeability rocks. This implies that in high-permeability rocks foam might not collapse abruptly at a single water saturation; instead, there is a range of water saturation over which foam weakens. In addition, we address the question of whether $P_{c}$ * is dependent on formation permeability. We estimate $P_{c} *$ from data for foam mobility versus foam quality and find, as did Khatib et al. (SPE Reservoir Eng., 1988, 3 (3), 919-926), who introduced the limiting capillary pressure concept, that $P_{\mathrm{c}}{ }^{*}$ can vary with permeability. It increases as permeability decreases, but not enough to reverse the trend of increasing foam apparent viscosity as permeability increases.

\section{INTRODUCTION}

Gas-injection enhanced oil recovery (EOR) projects often suffer from poor volumetric sweep because the density and viscosity of the injected gas are smaller than those of the oil under reservoir conditions. ${ }^{2,3}$ As a result, a large portion of the oil is not contacted by the injected gas and the ultimate recovery of the reservoir remains low. Foam can potentially solve this shortcoming by reducing gas mobility and increasing the viscous pressure drop in the flow direction that can act against gravity segregation. ${ }^{4-8}$ Foam also has the unique advantage that under some conditions it spontaneously reduces gas mobility more in high-permeability layers than in lowpermeability layers.

To predict the efficiency of a foam-displacement process on the field scale, foam models should reflect the most relevant physics of foam in porous media (e.g., the effects of permeability, surfactant concentration, oil saturation and composition, wettability, etc.). Several models have been proposed for modeling of foam in porous media, ${ }^{9-11}$ which can be divided into two main categories. Population-balance (PB) models ${ }^{12-16}$ attempt to describe the processes that create and destroy the lamellae that separate gas bubbles, as well as the effect of bubble size on gas mobility. The second group of models represents the effect of bubble size implicitly, through a mobility-reduction factor that depends on saturations, superficial velocities, and other factors. ${ }^{17-19}$ We call this second group "implicit-texture" (IT) models. PB models can be restructured to assume local equilibrium (LE) between the dynamics of lamella creation and destruction; all current IT models assume LE. These models contain adjustable parameters that are estimated by fitting the model to experiments. ${ }^{10,11,19-22}$ Several different foam models can fit the steady-state mobility of strong foam in a given porous medium to a satisfactory degree. ${ }^{11}$ To apply these models on the field scale, these parameters should be able to represent foam in the variety of geological strata in a reservoir, and in particular as a function of permeability.

Most foam models currently in use are built on the concept of the limiting capillary pressure, ${ }^{1}$ above which foam becomes unstable. The $P_{\mathrm{c}}$ * concept in porous media is analogous to the concept of the critical disjoining pressure in bulk foam, above which a single foam lamella breaks. ${ }^{23-25}$ Similarly, in foamdisplacement experiments in a porous medium, as gas saturation rises the local capillary pressure increases; at a certain point the capillary pressure is so great that foam films do not survive and the foam becomes coarser in texture. ${ }^{1}$

In an oil-free porous medium under steady-state conditions, foam exhibits two flow regimes depending on gas fractional

Received: February 2, 2015

Revised: April 8, 2015 

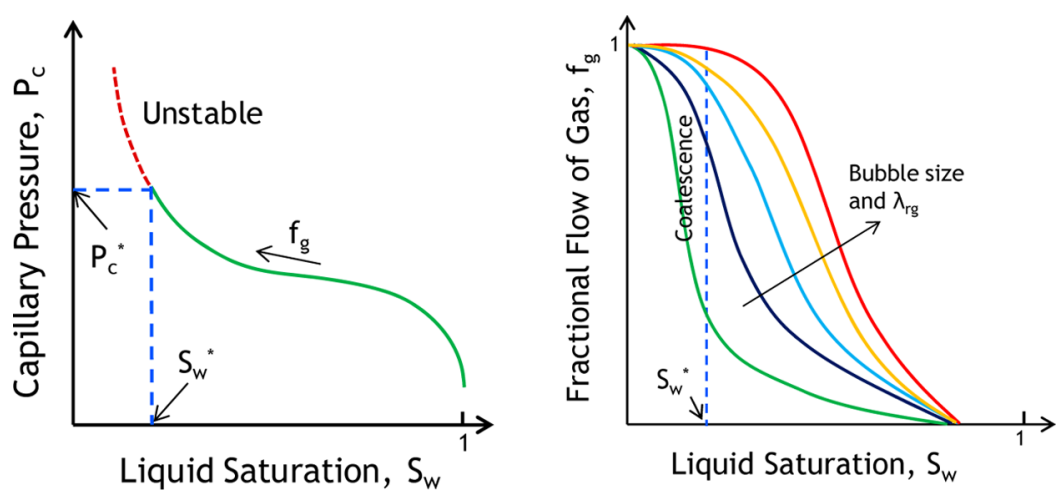

Figure 1. Limiting capillary pressure concept. In a porous medium, $P_{c}$ is a function of $S_{w}$. Meanwhile, the gas fractional-flow function depends on bubble size. At $P_{\mathrm{c}}{ }^{*}$, foam breaks. At this fixed water saturation, $S_{\mathrm{w}} * \equiv S_{\mathrm{w}}\left(P_{\mathrm{c}}^{*}\right)$, foam becomes coarser, mean bubble size increases, and gas fractional flow increases (adapted from Khatib et al. ${ }^{1}$ ). The LE behavior of foam would follow the red curve at small $S_{\mathrm{w}}$, then the dotted line, and then the green curve at large $S_{\mathrm{w}}$ on the right plot.
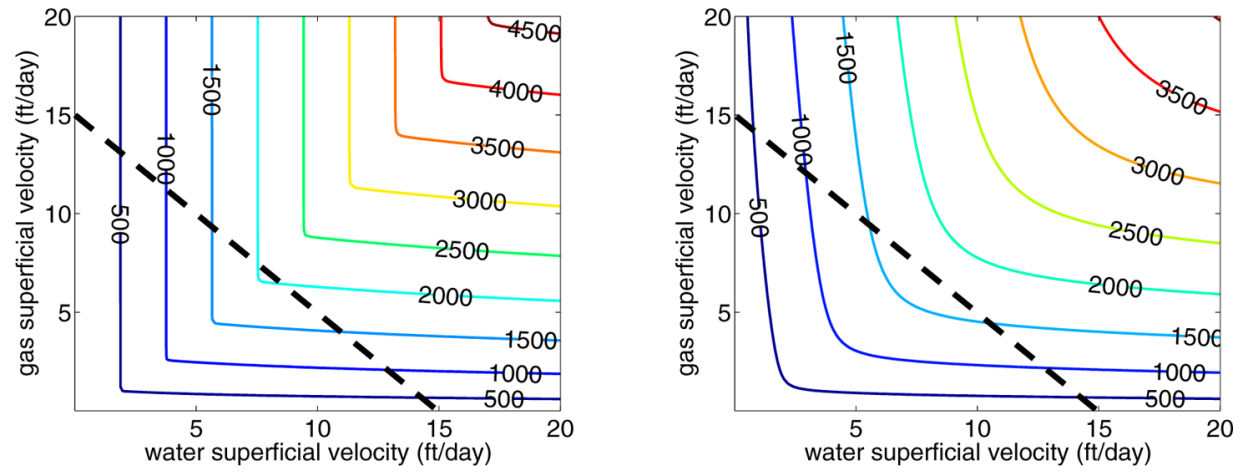

Figure 2. Pressure-gradient contours as a function of superficial velocities of gas and liquid calculated from the foam model presented in section 2 for epdry $=1 \times 10^{5}$ (left-hand panel) (abrupt transition between regimes) and epdry $=1 \times 10^{2}$ (right-hand plot) (less abrupt transition). The other parameters are kept constant $\left(\mathrm{fmmob}=4 \times 10^{4}\right.$; fmdry $=0.30$; fmcap $=2.46 \times 10^{-6}$; epcap $=0.30$; and other parameters in Table A.1 in Supporting Information). Foam quality increases as one moves from lower left to upper right. Also plotted (dotted line) is a hypothetical scan of foam quality at a fixed superficial velocity of $15 \mathrm{ft} /$ day.

flow (i.e., foam quality). ${ }^{26,27}$ If foam collapse is abrupt, the transition between regimes occurs at a single water saturation $S_{\mathrm{w}}{ }^{*}$ (the water saturation corresponding to $P_{\mathrm{c}}{ }^{*}$, see Figure 1); in the so-called high-quality regime, the pressure gradient along the core is independent of gas superficial velocity, while in the so-called low-quality regime, the pressure gradient is independent of liquid superficial velocity (Figure 2, left-hand panel). However, if foam collapse is not abrupt, there is a range of water saturations over which foam becomes coarser in texture. In this case, the contour plot of pressure gradient shows a rounded transition between regimes rather than a sharp corner (Figure 2, right-hand panel). For a constant total superficial velocity, the high-quality regime is the range of foam quality where the pressure gradient decreases with increasing foam quality, whereas in the low-quality regime the pressure gradient increases with increasing foam quality. By applying Darcy's law and treating foam as a single phase, an "apparent" viscosity of foam can be defined from the foam-induced pressure gradient.

$$
\mu_{\mathrm{f}}^{\mathrm{app}} \equiv \frac{k \nabla P}{u_{\mathrm{t}}}
$$

where $\nabla P$ is the magnitude of pressure gradient, $k$ permeability, and $u_{\mathrm{t}}$ total superficial velocity. The apparent viscosity of foam, or equivalently, the pressure gradient, is at a maximum where the line of constant total superficial velocity crosses the transition on a plot similar to Figure 2.

The limiting capillary pressure is believed to vary with surfactant type and concentration, electrolyte concentration, gas flow rate, and porous medium permeability. ${ }^{1}$ Nonetheless, the exact dependence on these variables is not known. Our main objective here is to explore the dependency of the limiting capillary pressure on permeability, surfactant type, and surfactant concentration. We use an IT foam model and examine its capacity to fit steady-state foam mobility in porous media. The permeability dependence of the parameters in the IT model will also be examined. To that end we will use some published steady-state foam data. These experiments have been conducted with rocks with different permeability and using different surfactant solutions. The structure of the paper is as follows. First, in section 2, we describe the IT foam model and briefly explain methods to extract its relevant parameters. Next, in section 3, we fit model parameters to steady-state foam data and establish the permeability dependence of the parameters. In section 4, the derived parameters are used to evaluate the relationship between rock permeability and the limiting capillary pressure. The effects of surfactant type and concentration on $P_{c}^{*}$ are also discussed. 


\section{IMPLICIT-TEXTURE FOAM MODEL}

We focus our study on one steady-state implicit-texture foam model, that in STARS. ${ }^{9,10,22,28}$ In that model gas mobility is scaled by a function, FM, when foam is present:

$$
\lambda_{\mathrm{g}}^{\mathrm{f}}=\lambda_{\mathrm{g}}^{\mathrm{nf}} \mathrm{FM}=\frac{\lambda_{\mathrm{g}}^{\mathrm{nf}}}{1+\mathrm{fmmob} \prod_{i=1}^{n} F_{i}}
$$

where $\lambda_{\mathrm{g}}$ is gas mobility and superscripts $\mathrm{f}$ and $\mathrm{nf}$ represent the cases with and without foam, respectively. Parameter fmmob is the maximum (or reference) mobility reduction factor that could be achieved by foam when all conditions are favorable. The " $F$ " functions in eq 2 reflect the effects of different physical parameters, such as surfactant concentration, water saturation, oil saturation (and composition), capillary number, etc., on foam behavior in porous media. In this paper we focus on the dry-out and shear-thinning functions, which are elements of the $F_{i}$ functions. The dry-out function is defined in STARS as

$$
F_{\mathrm{w}}=0.5+\frac{1}{\pi} \arctan \left(\operatorname{epdry}\left(S_{\mathrm{w}}-\text { fmdry }\right)\right)
$$

Parameter fmdry is the water saturation at which foam experiences significant coalescence, and consequently in the limit of large epdry it is equivalent to $S_{\mathrm{w}}{ }^{*}$. Parameter epdry controls the sharpness of transition from the high-quality regime to the low-quality regime: when epdry is very large, the transition is sharp and foam collapses within a very narrow range of water saturation. When epdry approaches infinity, foam coalescence occurs at a single water saturation $\left(S_{\mathrm{w}}{ }^{*}\right)$. In the most recent version of STARS, the parameter fmdry is renamed sfdry, and epdry is renamed sfbet. ${ }^{29}$ In that model, sfdry can be represented as a function of surfactant concentration, oil saturation, salt concentration, and capillary number. If one disables these other functionalities, sfdry is a constant and plays the same role as fmdry does above.

The shear-thinning function is defined as

$$
F_{\text {shear }}=\left\{\begin{array}{cc}
1 & N_{\mathrm{Ca}}\left(=\frac{k \nabla P}{\sigma}\right)<\text { fmcap } \\
\left(\frac{\text { fmcap }}{N_{\mathrm{Ca}}}\right)^{\text {epcap }} & N_{\mathrm{Ca}}\left(=\frac{k \nabla P}{\sigma}\right)>\text { fmcap }
\end{array}\right.
$$

where $\nabla P$ is the magnitude of pressure gradient, $k$ permeability, and $\sigma$ the surface tension. Parameter fmcap should be set to the smallest capillary number expected to be encountered by foam in the simulation, ${ }^{19,20}$ and epcap represents the extent of shear-thinning behavior. Newtonian behavior corresponds to epcap $=0$, and positive epcap corresponds to shear-thinning behavior. To obtain epcap from experimental data, we use the definition of apparent foam viscosity

$$
\mu_{\mathrm{f}}^{\mathrm{app}}=\frac{1}{\frac{k_{\mathrm{rw}}\left(S_{\mathrm{w}}\right)}{\mu_{\mathrm{w}}}+\frac{k_{\mathrm{rg}} \mathrm{nf}\left(S_{\mathrm{w}}\right)}{\mathrm{FM} \mu_{\mathrm{g}}}}
$$

where $u_{\mathrm{t}}$ is total superficial velocity, $k_{\mathrm{rw}}$ water relative permeability, and

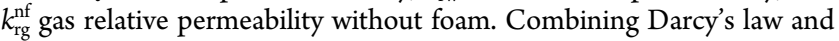
eq 5 , we obtain

$$
\nabla P=\frac{u_{\mathrm{t}}}{k} \frac{1}{\frac{k_{\mathrm{rw}}\left(S_{\mathrm{w}}\right)}{\mu_{\mathrm{w}}}+\frac{k_{\mathrm{rg}}^{\mathrm{nf}}\left(S_{\mathrm{w}}\right)}{\mu_{\mathrm{g}}\left(1+\mathrm{fmmob} \cdot F_{\mathrm{w}} \cdot\left(\frac{\text { fmcap }}{k \nabla P / \sigma}\right)^{\text {epcap }}\right)}}
$$

Procedures to fit data for $\mu_{\mathrm{f}}^{\text {app }}$ as a function of foam quality to the IT foam model have been provided by several authors. We have modified the procedure explained by $\mathrm{Ma}$ et al. ${ }^{10,22}$ by adding a shear-thinning function because in their approach smaller values can be assigned to epdry, although as shown later choosing very large values of epdry ${ }^{19,20}$ does not alter the general conclusions of this paper. The procedure used here, described in Appendix B in Supporting Information, seeks a least-squares fit to all the $\mu_{\mathrm{f}}^{\text {app }}$ data subject to preset weighting and constraints.

\section{DEPENDENCE OF FOAM MODEL PARAMETERS ON PERMEABILITY}

We apply the data reported by Moradi-Araghi et al. ${ }^{30}$ to investigate the effect of rock permeability on foam model parameters. The experiments were conducted with $\mathrm{CO}_{2}$ at 98 ${ }^{\circ} \mathrm{F}$ and 2000 psi with reservoir-core material of different permeabilities. The cores were $1 \mathrm{in}$. in diameter and $4.84 \mathrm{in}$. in length. The rocks were of similar type and belonged to the same unit from the West Cowden reservoir. The overall pressure drop in each foam experiment was divided by that for water injection at $S_{\mathrm{w}}=1$ and reported as reduction factor (RF). Therefore, to calculate the apparent viscosity of foam in $\mathrm{cp}$ as a function of gas fraction or foam quality $\left(f_{\mathrm{g}}\right)$, the reported reduction factors were multiplied by water viscosity at experimental conditions:

$$
\mu_{\mathrm{app}}^{\mathrm{f}}\left(f_{\mathrm{g}}\right)=\operatorname{RF}\left(f_{\mathrm{g}}\right) \cdot \mu_{\mathrm{w}}=0.65 \mathrm{RF}\left(f_{\mathrm{g}}\right)[\mathrm{cp}]
$$

Thus, the apparent viscosity, $\mu_{\mathrm{f}}^{\text {app }}$, is the viscosity one would infer for foam at the given fractional flow if foam were treated as a single-phase fluid (see eqs 1 and 5).

This data set is unusually valuable in presenting the behavior of a single foam formulation over a range of foam qualities in media of widely differing permeability. However, there is some concern that the entrance effect ${ }^{31}$ might affect these data. In particular, if the entrance length (distance from the inlet over which foam comes to full strength) varies systematically as a function of permeability, that would appear in our data as an effect of permeability on IT foam parameters. We present these results as the best available to date, pending results for longer cores that exclude the entrance effect.

Figure 3 presents the gas apparent viscosity as a function of foam quality for rocks with different permeabilities. The

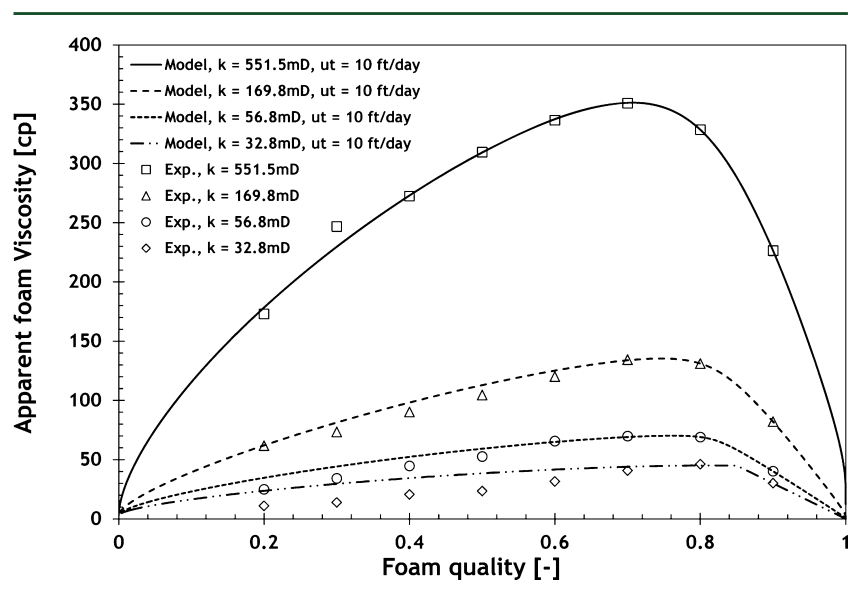

Figure 3. Apparent viscosity of $\mathrm{CO}_{2}$ foam made with $2000 \mathrm{ppm}$ of Chaser CD-1050 as a function of permeability.

symbols are the experimental data, and the lines are calculated using eqs 2 and 5 with the parameters listed in Table 1 and Table A.1 in Supporting Information. At all foam qualities, the apparent viscosity of the gas increases with increasing permeability, i.e., foam appears to be stronger in the rock with higher permeability. Moreover, the existence of two regimes is clear for all permeabilities; the apparent viscosity of foam increases and reaches a maximum as the foam quality 
Table 1. Parameter Estimation with Permeability-Dependent epdry for Foam Data in Figure 3

\begin{tabular}{|c|c|c|c|c|c|c|c|c|}
\hline \multirow[b]{2}{*}{$\begin{array}{l}\text { permeability } \\
(\mathrm{mD})\end{array}$} & \multirow[b]{2}{*}{$\begin{array}{c}\text { total velocity (ft/ } \\
\text { day) }\end{array}$} & \multicolumn{7}{|c|}{$n_{\mathrm{w}}=4$} \\
\hline & & fmmob & epdry & fmdry & fmcap & epcap & $\begin{array}{c}\text { fmmob } F_{5} \\
\nabla P=10 \mathrm{MPa} / \mathrm{m}\end{array}$ & $\begin{array}{c}\text { fmmob } F_{5} \\
\nabla P=0.1 \mathrm{MPa} / \mathrm{m}\end{array}$ \\
\hline 551.5 & 20 & 25171.2 & 80.6 & 0.265 & $2.46 \times 10^{-05}$ & 0.242 & 14871.43 & 29400.36 \\
\hline 551.5 & 5 & 17997.5 & 80.6 & 0.265 & $2.46 \times 10^{-05}$ & 0.242 & 18349.3 & 17775.4 \\
\hline 551.5 & 10 & 21284.2 & 80.6 & 0.265 & $2.46 \times 10^{-05}$ & 0.242 & 8479.31 & 25844.04 \\
\hline 169.8 & 10 & 2058.6 & 195.9 & 0.308 & $2.46 \times 10^{-05}$ & $2.24 \times 10^{-14}$ & 2058.6 & 2058.6 \\
\hline 56.8 & 10 & 899.4 & $>530$ & 0.349 & $2.46 \times 10^{-05}$ & $2.24 \times 10^{-14}$ & 899.4 & 899.4 \\
\hline 32.8 & 10 & 502.3 & $>10000$ & 0.367 & $2.46 \times 10^{-05}$ & $4.33 \times 10^{-14}$ & 502.3 & 502.3 \\
\hline
\end{tabular}

Table 2. Parameter Estimation Assuming a Very Large epdry of 100000 for Foam Data in Figure 3

\begin{tabular}{|c|c|c|c|c|c|c|}
\hline \multirow[b]{2}{*}{ permeability $(\mathrm{mD})$} & \multirow[b]{2}{*}{ total velocity (ft/day) } & \multicolumn{5}{|c|}{$n_{\mathrm{w}}=4$} \\
\hline & & fmmob & epdry & fmdry & fmcap & epcap \\
\hline 551.5 & 10 & 47434 & $1.00 \times 10^{5}$ & 0.261 & $2.46 \times 10^{-5}$ & 0.449 \\
\hline 169.8 & 10 & 1964.5 & $1.00 \times 10^{5}$ & 0.308 & $2.46 \times 10^{-5}$ & $2.22 \times 10^{-14}$ \\
\hline 56.8 & 10 & 886.2 & $1.00 \times 10^{5}$ & 0.349 & $2.46 \times 10^{-5}$ & $3.4 \times 10^{-14}$ \\
\hline 32.8 & 10 & 502.3 & $1.00 \times 10^{5}$ & 0.367 & $2.46 \times 10^{-5}$ & $4.33 \times 10^{-14}$ \\
\hline
\end{tabular}

increases from 0 (low-quality regime) and thereafter decreases with further increase in foam quality (high-quality regime).

To extract foam model parameters from experimental data, we assume all core plugs have similar wetting behavior and consequently fix the value of the Corey exponent in the water relative-permeability function to $n_{\mathrm{w}}=4$ (see eq C.4 in Supporting Information). Foam model parameters are sensitive to relative-permeability parameters, and in particular $n_{\mathrm{w}}$ can greatly impact the parameters. ${ }^{10}$ In the following, we test sensitivity of our results to $n_{\mathrm{w}}$. The total superficial velocity of the experiments, which is needed for the calculation of capillary number, is not given in the paper. We assume a value of $10 \mathrm{ft} / \mathrm{d}$ $\left(3.5 \times 10^{-5} \mathrm{~m} / \mathrm{s}\right)$ in our calculations. However, the total superficial velocity used in the calculations affects only the value of fmmob, and the other parameters remain the same if a different value is used, albeit by fixing the value of fmcap. The model parameter set in Table 1 includes a fit allowing all parameters to vary (except for fmcap, which is simply a reference capillary number for $F_{5}$ in eq 2) and constraining epcap $>0$ (i.e., not allowing for shear-thickening in the lowquality regime. The model fits using different constraints are shown in Table A.2 in Supporting Information.

In all the data fits, the value of fmdry decreases with increasing permeability. This is an indication that foam reduces water saturations to lower values as permeability increases. In fact, the values of fmdry are remarkably similar for all model fits, as shown in Table A.2 in Supporting Information. Moreover, the fitted values of epcap indicate that foam exhibits shear-thinning behavior, which becomes more pronounced as permeability of the rock increases. The model fit using the approach of Boeije and Rossen, ${ }^{20}$ i.e., using very large epdry values, supports this conclusion as well (see Table 2 ).

Foam exhibits shear-thickening behavior in the lowestpermeability rocks for the experimental data assessed in this paper. We know of no physical justification for this behavior.

Direct comparison of values of fmmob are ambiguous; if behavior is non-Newtonian in the low-quality regime, the value of fmmob is affected by the arbitrary choice of the reference state represented by fmcap. ${ }^{20} \mathrm{~A}$ better comparison is that for $\left[\mathrm{fmmob} \cdot \mathrm{F}_{5}\right]$ at a fixed pressure gradient. This represents the mobility reduction in the low-quality regime at the fixed pressure gradient. In Table 1 and Table A.2 we show values of $\left[\mathrm{fmmob} \cdot F_{5}\right]$ at a pressure gradient of $10 \mathrm{MPa} / \mathrm{m}$, which is representative of a pressure gradient seen in all the corefloods in this paper, and at $0.1 \mathrm{MPa} / \mathrm{m}$, which would be more representative of field application. The value of this product at any pressure gradient is given by

$$
\mathrm{fmmob} \cdot F_{5}=\mathrm{fmmob}\left(\frac{\text { fmcap }}{k \nabla p / \sigma}\right)^{\text {epcap }}
$$

The finding that the product $\left[\mathrm{fmmob} \cdot \mathrm{F}_{5}\right]$ increases with increasing permeability is surprising. This product represents the mobility reduction for wet foams in the low-quality regime. It does not reflect the effect of capillary pressure on foam, which makes foam stronger in high-permeabilities in the highquality regime. ${ }^{1}$ The bubbles in the low-quality regime are thought to be about the size of pores. ${ }^{27,32}$ Because pores are expected to be smaller in the lower-permeability media, there are more lamellae resisting flow per unit length in these media. The total resistance is a function of many factors, including gas trapping and the resistance to individual lamellae as they flow; none of these immediately suggest lower resistance to flow in lower-permeability media. If confirmed by further study, this paradox deserves further investigation.

The assumption of a very large epdry value for all experiments does not lead to a good match in the high-quality regime, particularly for the high-permeability rock. An example is shown in Figure 4, on which we plot the curves obtained with different epdry values and compare it to the experimental data. The convex trend observed in the high-quality regime in the experiment can be best matched with selecting epdry $=80.6$. This implies that, for the set of data examined in this paper, the transition from high-quality to low-quality is not abrupt and foam does not collapse at a constant water saturation. For small epdry values, the transition water saturation $\left(S_{\mathrm{w}}{ }^{\mathrm{tr}}\right)$ is different than the limiting water saturation $\left(S_{\mathrm{w}}{ }^{*}\right)$, which suggests that water fractional-flow function $\left(f_{\mathrm{w}}\right)$ decreases smoothly from $S_{\mathrm{w}}{ }^{\text {tr }}$ to shock water saturation. Moreover, we notice from Table 1 as the permeability of rock decreases, the epdry value increases. For low permeability cores, the match can be obtained by choosing epdry $>10000$, implying abrupt transition. 


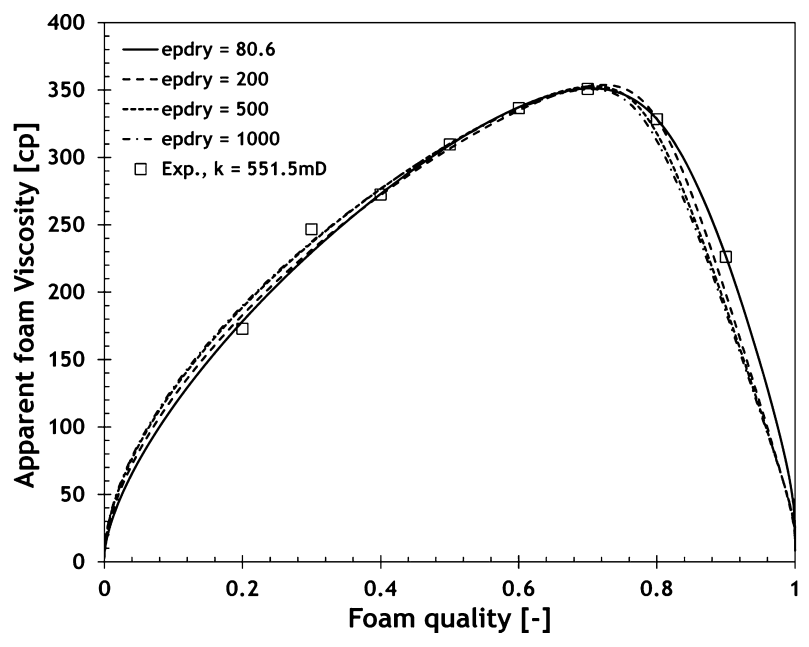

Figure 4. Results of the model with different epdry values compared to the experimental data.

If we neglect the curved behavior of foam in the high-quality regime, it is possible to fit the data with a single or permeabilityindependent large epdry value. The parameters are listed in Table 2. Note that with change of epdry, the other parameter should also be adjusted.

Figure 5 shows a second set of experimental data for a different surfactant formulation and compares them to the

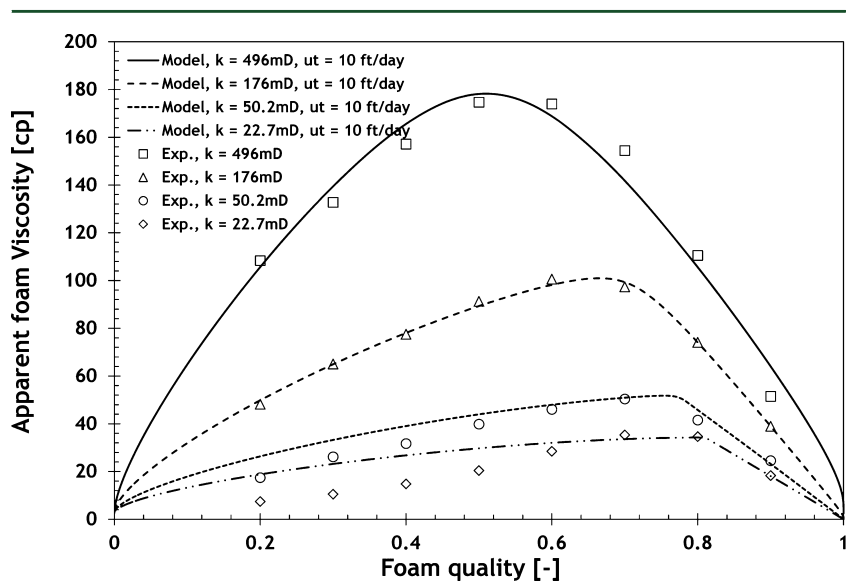

Figure 5. Apparent viscosity of $\mathrm{CO}_{2}$ foam made with $2000 \mathrm{ppm}$ foamer NES-25 as a function of permeability.

model. Again, epdry decreases with increasing permeability in Table 3. Once again, foam exhibits shear-thinning behavior in the rock with high permeability. This conclusion, that epdry decreases with increasing permeability, needs to be confirmed by further study, as Kapetas et al. ${ }^{33}$ found no correlation between epdry and permeability in their experiments. Moreover, the original study of the limiting capillary pressure, ${ }^{1}$ which reported an abrupt transition between regimes, was conducted in beadpacks of enormous permeability (up to 10000 Darcy).

Our main interest in this paper is to investigate the effect of the permeability on $P_{\mathrm{c}}{ }^{*}$, which is reflected in fmdry; as mentioned, its value is relatively independent of the fitting procedure. The next section addresses this issue.

\section{EFFECT OF PERMEABILITY ON LIMITING CAPILLARY PRESSURE}

The general form of the gas-water capillary pressure function can be expressed as ${ }^{34-37}$

$$
P_{\mathrm{c}}=P_{\mathrm{c} @ S_{\mathrm{wc}}}\left(1-b S_{\mathrm{wD}}\right)^{-1 / \lambda}
$$

where $P_{\mathrm{c} @ S_{\mathrm{wc}}}$ is the capillary pressure at the connate water saturation, $S_{\mathrm{wc}}$ when drainage capillary pressure is used. $S_{\mathrm{wD}}$ is the normalized water saturation defined as

$$
S_{\mathrm{wD}}=\frac{S_{\mathrm{w}}-S_{\mathrm{wc}}}{1-S_{\mathrm{wc}}-S_{\mathrm{gc}}}
$$

Parameter $b$ is a constant and can be calculated from

$$
b=1-\left(\frac{P_{\mathrm{ce}}}{P_{\mathrm{c} @ S_{\mathrm{wc}}}}\right)^{-\lambda}
$$

where $P_{\text {ce }}$ is the entry capillary pressure of the porous medium and $\lambda$ is the sorting factor. For a known value of $S_{\mathrm{w}}{ }^{*}$, the value of limiting capillary pressure, $P_{\mathrm{c}}{ }^{*}$, can be estimated using eq 9 . If the wetting states of the rocks are similar, the scaling of capillary pressure with permeability can be estimated using the Leverett J-function:

$$
J\left(S_{\mathrm{w}}\right)=C_{\mathrm{J}} \frac{P_{\mathrm{c}}^{k}\left(S_{\mathrm{w}}\right)}{\sigma} \sqrt{\frac{k}{\phi}}
$$

where $C_{\mathrm{J}}$ is a constant. Therefore, to understand the effect of permeability on the limiting capillary pressure, the J-Leverett function was calculated from capillary pressure data for a Berea sandstone core with permeability of about $500 \mathrm{mD} .^{38}$ The red curve in Figure 6 is obtained by using $P_{\mathrm{ce}}=2 \mathrm{kPa}$ and $P_{\mathrm{c} @ S_{\mathrm{wc}}}$ $=1000 \mathrm{kPa}$ for $k=551 \mathrm{mD}$, and the relative-permeability parameters in Table A.1 in Supporting Information. The capillary pressure curves of the other permeabilities were calculated using eq 12 . Note that in this model connate water saturation is assumed to be at $P_{\mathrm{c} @ S_{w c}}=1000 \mathrm{kPa}$; and as a result the connate water saturation is also permeability-dependent (as indicated in Figure 6).

Once the capillary pressures are known, taking the value of fmdry (extracted from experiments) as the limiting water saturation, the limiting capillary pressure can be estimated for each set of foam data for different permeability rocks. Figure 7 shows the relation between $P_{c}{ }^{*}$ and rock permeability. The

Table 3. Parameter Estimation for Foam Data in Figure 5

\begin{tabular}{ccccccc} 
& & \multicolumn{3}{c}{$n_{\mathrm{w}}=4$} \\
\cline { 3 - 7 } permeability $(\mathrm{mD})$ & total velocity $(\mathrm{ft} /$ day $)$ & fmmob & epdry & fmdry & fmcap & epcap \\
496 & 10 & 4386 & 89.8 & 0.334 & $2.46 \times 10^{-05}$ & $2.22 \times 10^{-14}$ \\
176 & 10 & 1566 & 358.3 & 0.348 & $2.46 \times 10^{-05}$ & $2.22 \times 10^{-14}$ \\
50.2 & 10 & 620 & $>5000$ & 0.380 & $2.46 \times 10^{-05}$ & $2.32 \times 10^{-14}$ \\
22.7 & 10 & 364 & $>10000$ & 0.397 & $2.46 \times 10^{-05}$ & $3.94 \times 10^{-13}$
\end{tabular}




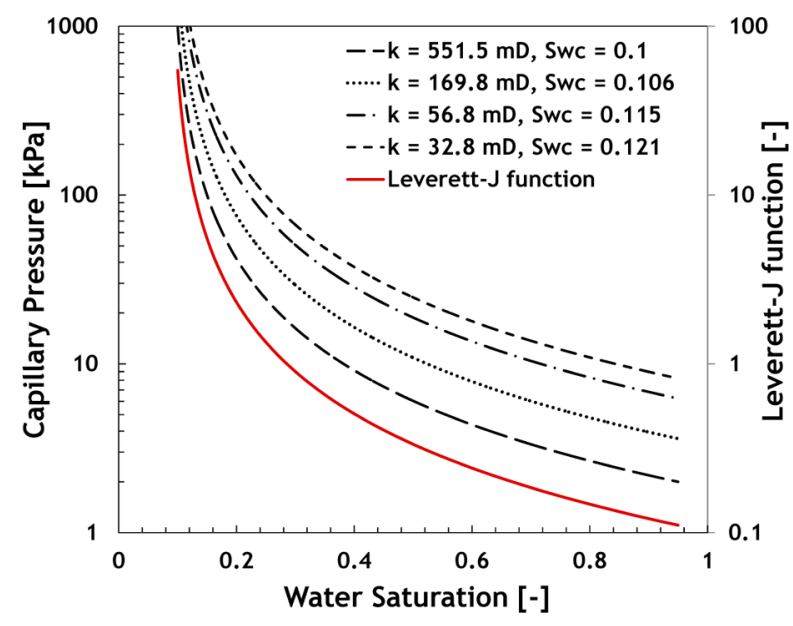

Figure 6. Calculated capillary pressure curves for rocks with different permeability. $P_{\mathrm{ce}}(k=551 \mathrm{md})=2 \mathrm{kPa} ; P_{\mathrm{c} @ S_{\mathrm{wc}}}=1000 \mathrm{kPa}$; and $\lambda=2 /$ 3.

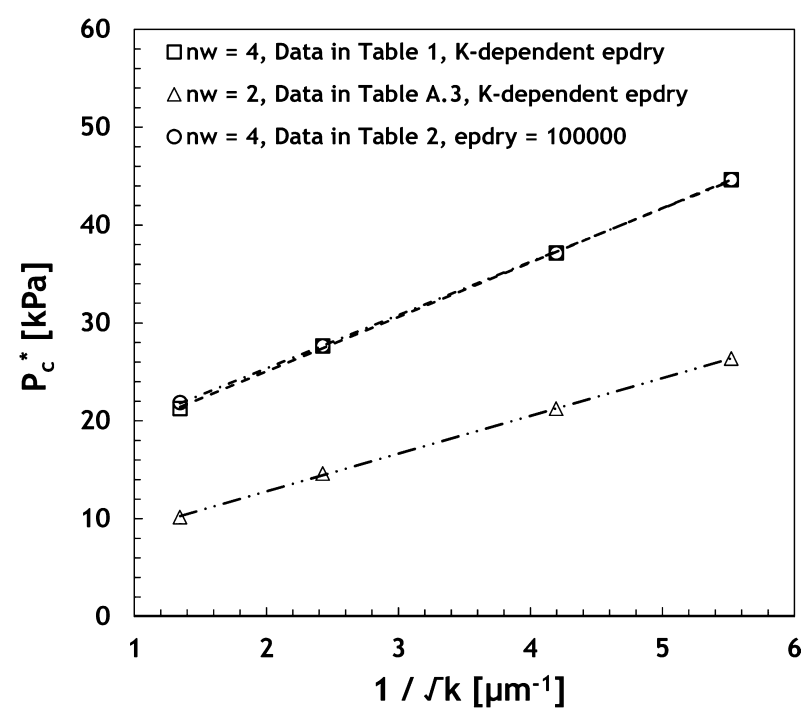

Figure 7. Effect of permeability on the limiting capillary pressure. The points are extracted using parameters in Tables 1 and 2 .

value of limiting capillary pressure increases as permeability decreases. Moreover, $P_{c}{ }^{*}$ varies linearly with $1 /(k)^{1 / 2}$. It is interesting to note that this scaling is independent of the value of epdry and choice of $n_{\mathrm{w}}$ in our model fits, as shown in Figure 7. To extract the data for $n_{\mathrm{w}}=2$, we assumed that capillary pressure and relative permeability parameters can be related as described in Appendix $\mathrm{C}$ in Supporting Information. ${ }^{44}$ In all cases the value of $P_{\mathrm{c}} *$ increases as $n_{\mathrm{w}}$ increases.

A trend of decreasing $P_{\mathrm{c}}{ }^{*}$ with increasing permeability can also be observed between the direct measurements of $P_{c} *$ and rock permeability of Khatib et al. ${ }^{1}$ (Figure 8 ). Rossen and Zhou ${ }^{39}$ came to a similar conclusion based on inferences from limited data, as did Rossen and $\mathrm{Lu}^{40}$ based on a wide survey of data (but not taking account of the distinction between the two foam flow regimes). This finding implies that $P_{c} *$ for foam in porous media depends not only on disjoining pressure but also on the permeability of the porous medium. Although $P_{\mathrm{c}}{ }^{*}$ increases somewhat with decreasing permeability, the trend is still that foam is stronger (lower gas relative mobility) in rocks with greater permeability, as shown in Figures 3 and 5 .

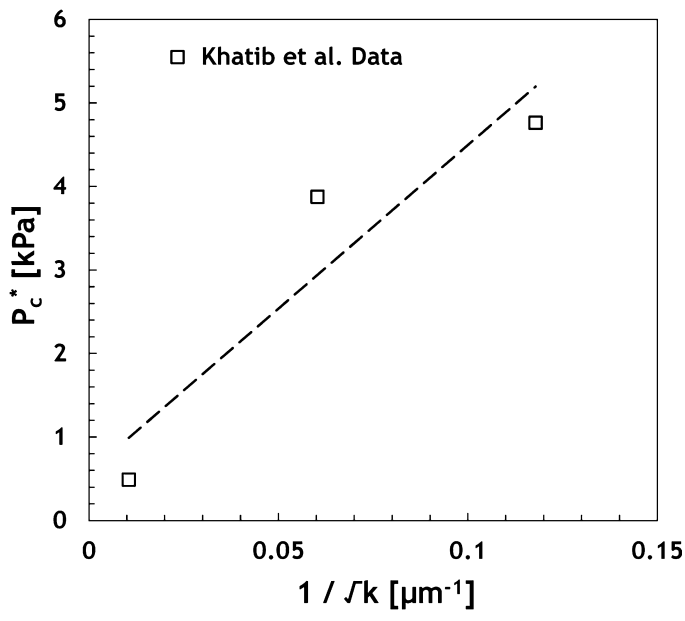

Figure 8. Limiting capillary pressure measured by Khatib et al. ${ }^{1}$ as a function of permeability.

Decreasing $P_{\mathrm{c}}{ }^{*}$ with increasing permeability implies diversion from a lower-permeability into a higher-permeability layer if layers are in capillary equilibrium. ${ }^{39,40}$ Rossen and $\mathrm{Lu}^{40}$ found, though, that capillary equilibrium is difficult to achieve with foam. Rossen et al. ${ }^{41}$ report that, at short times, foam diverts flow from high- to low-permeability layers; at much longer times, capillary pressure gradients may drive water out of the higher-permeability layer and weaken foam there, especially if the viscous pressure gradient is modest.

As mentioned earlier, a premise of the of the "limitingcapillary-pressure" concept is that the stability of static foam film or lamella is limited by critical capillary pressure, which is equal to the critical disjoining pressure. Dynamic systems can expect to have a limiting capillary pressure that is less than this critical capillary pressure because the foam lamella stretches as a foam lamella moves from a pore throat to a pore body; thus, its area increases. ${ }^{42}$ If the lamella cannot recruit sufficient water from the Plateau border, the lamella must thin to compensate for the increase in area, i.e., to maintain constant volume. If the lamella can recruit water from the Plateau border, it does not thin as much. As the lamella thins, the disjoining pressure increases. If the lamella thins to the critical disjoining pressure, it becomes unstable and breaks. As the lamella velocity increases, the limiting capillary pressure must decrease to compensate for the increase in disjoining pressure as the lamella stretches in passing from a pore throat to a pore body. As the rock permeability increases, the limiting capillary pressure can be expected to decrease because area of the lamella increases relative to the volume of water in the Plateau border. The Plateau border is the perimeter of the lamella and thus proportional to the characteristic dimension, whereas the lamella area is proportional to the square of the characteristic dimension. Therefore, as permeability increases, there is less water, relatively speaking, nearby in the Plateau border to stabilize the lamella as it stretches.

\section{EFFECT OF PROPERTIES OF SURFACTANT SOLUTION ON LIMITING CAPILLARY PRESSURE}

The disjoining pressure depends on surfactant type and concentration, among other parameters. Foam films with greater disjoining pressure can survive a greater imposed capillary pressure. We observe from Figure 9 that surfactant type has also an influence on the strength of foam in porous 


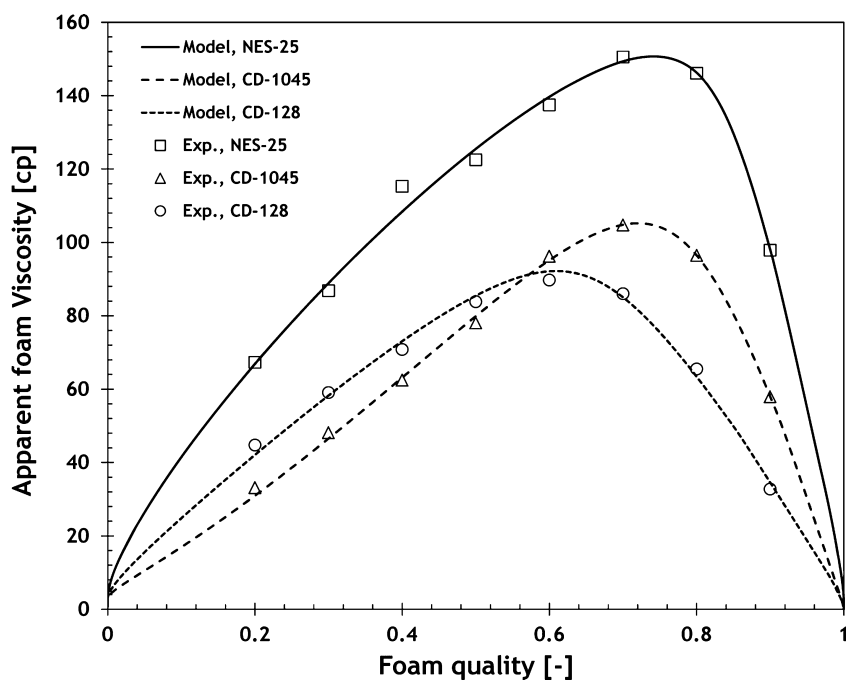

Figure 9. $\mathrm{CO}_{2}$ foam data with different surfactant types in a core with permeability of $176 \mathrm{md}$ (Moradi-Araghi et al. ${ }^{30}$ ). The concentration of surfactant in each test was set to $2000 \mathrm{ppm}$.

media. The foam data in Figure 9 were fitted to the STARS model with the procedure explained in Appendix B in Supporting Information, and using the fitted fmdry values, the magnitude of $P_{c} *$ was estimated for each surfactant. These parameters are presented in Table 4 . In addition, using the data reported by $\mathrm{Ma}$ et $\mathrm{al}^{2}{ }^{22}$ who studied the effect of surfactant concentration on foam in sandpacks, we find a trend of increasing $P_{c}$ * with increasing surfactant concentration (Table 5). The smaller values of the $P_{c} *$ values in Table 5 compared to those reported in Figure 7 are due to very high permeability of the rock used by $\mathrm{Ma}$ et al. ${ }^{22}$ experiments. A similar trend was reported by Apaydin and Kovscek. ${ }^{43}$

\section{CONCLUSIONS}

An implicit-texture foam model, specifically the STARS model examined in this study, can represent the effects of rock permeability and surfactant type and concentration on steadystate foam flow in porous media.

The fit to experimental data suggests that all foam model parameters are permeability-dependent. This becomes important when the effect of heterogeneity is considered in field scale.

For the experimental data investigated in this paper, the value of epdry increases as rock permeability decreases. These data suggest that, for certain formulations in high permeability rocks, foam does not collapse abruptly at a single fixed water saturation. Rather, there is a range of water saturations over which foam weakens. The width of this range narrows with decreasing permeability in the data examined.

For the cases examined, the limiting capillary pressure, $P_{c}$ *, increases as permeability decreases, but not enough to reverse the trend of decreasing foam apparent viscosity as permeability decreases. Foam is still stronger in higher-permeability rock.
Table 5. Effect of Surfactant Concentration on Limiting Capillary Pressure ${ }^{a}$

$\begin{array}{cc}\text { surfactant concentration }(\mathrm{wt} \%) & P_{\mathrm{c}}^{*}(\mathrm{kPa}) \\ 1 & 1 \\ 0.5 & 0.5 \\ 0.02 & 0.45\end{array}$

${ }^{a}$ Data from Ma et al. ${ }^{22}$

\section{ASSOCIATED CONTENT}

\section{S Supporting Information}

Parameters of fractional flow function and foam model, details of fitting procedure, and details regarding relation between capillary and relative permeability. The Supporting Information is available free of charge on the ACS Publications website at DOI: 10.1021 /acs.energyfuels.5b00248.

\section{AUTHOR INFORMATION}

Notes

The authors declare no competing financial interest.

\section{ACKNOWLEDGMENTS}

This paper is dedicated to Zara Khatib, who was the first to measure limiting capillary pressure for foam in porous media. ${ }^{1}$ The authors thank Shell Global Solutions International for their support and permission to publish this work.

\section{REFERENCES}

(1) Khatib, Z. I.; Hirasaki, G. J.; Falls, A. H. Effects of capillary pressure on coalescence and phase mobilities in foams flowing through porous media. SPE Reservoir Eng. 1988, 3 (3), 919-926.

(2) Lake, L. W. Enhanced Oil Recovery. Prentice Hall: Englewood Cliffs, NJ, 1989.

(3) Bedrikovetsky, P. G. Mathematical theory of oil \& gas recovery (with applications to ex-USSR oil \& gas condensate fields). Kluwer Academic Publishers: London, 1993.

(4) Kovscek, A. R.; Radke, C. J. Fundamentals of Foam Transport in Porous Media. In Foams: Fundamentals and Applications in the Petroleum Industry; Schramm, L. L., Ed.; ACS Advances in Chemistry Series No. 242; American Chemical Society: Washington, DC, 1994; Chapter 3, pp 115-163.

(5) Rossen, W. R. Foams in Enhanced Oil Recovery. In Foams: Theory, Measurements and Applications;Prud'homme, R. K., Khan, S., Eds.; Marcel Dekker: New York, 1996.

(6) Hirasaki, G. J.; Lawson, J. B. Mechanisms of foam flow in porous media: Apparent viscosity in smooth capillaries. SPE J. (Soc. Pet. Eng.) 1985, 25 (2), 176-190.

(7) Hirasaki, G. J. The steam foam process. J. Pet. Technol. 1989, 41 (5), 449-456.

(8) Farajzadeh, R.; Andrianov, A.; Zitha, P. L. J. Investigation of immiscible and miscible foam for enhancing oil recovery. Ind. Eng. Chem. Res. 2010, 49 (4), 1910-1919.

(9) Farajzadeh, R.; Andrianov, A.; Krastev, R.; Hirasaki, G.; Rossen, W. R. Foam-oil interaction in porous media: Implications for foamassisted enhanced oil recovery. Adv. Colloid Interface Sci. 2012, 183$184,1-13$.

Table 4. Estimation of Foam Parameters for Data in Figure 9

\begin{tabular}{|c|c|c|c|c|c|c|}
\hline \multirow[b]{2}{*}{ surfactant } & \multicolumn{6}{|c|}{$n_{\mathrm{w}}=4$} \\
\hline & fmmob & fmdry & epdry & fmcap & epcap & $\mathrm{P}_{\mathrm{c}}^{*}(\mathrm{kPa})$ \\
\hline NES-25 & 1955.2 & 0.298 & 123.3 & $2.46 \times 10^{-5}$ & -0.053 & 28.8 \\
\hline CD-1045 & 209.1 & 0.324 & 123.3 & $2.46 \times 10^{-5}$ & -0.595 & 23.9 \\
\hline CD-128 & 631.27 & 0.362 & 113 & $3.88 \times 10^{-5}$ & -0.320 & 19.5 \\
\hline
\end{tabular}


(10) Ma, K.; Farajzadeh, R.; Lopez-Salinas, J. L.; Miller, C. A.; Biswal, S. L.; Hirasaki, G. J. Non-uniqueness, numerical artifacts, and parameter sensitivity in simulating steady-state and transient foam flow through porous media. Transp. Porous Media 2014, 102, 325348.

(11) Lotfollahi, M.; Farajzadeh, R.; Varavei, A. J.; Delshad, M.; Rossen, W. R. Comparison of Population balance and Local Equilibrium Foam Models. J. Pet. Sci. Eng., 2015, under review.

(12) Falls, A. H.; Hirasaki, G. J.; Patzek, T. W.; Gauglitz, D. A.; Miller, D. D.; Ratulowski, T. Development of a mechanistic foam simulator: The population balance and generation by snap-Off. SPE Reservoir Eng. 1988, 3 (3), 884-892.

(13) Friedmann, F. C.; Chen, W. H.; Gauglitz, P. A. Experimental and simulation study of high-temperature foam displacement in porous media. SPE Reservoir Eng. 1991, 6 (1), 37-45.

(14) Kovscek, A. R.; Patzek, T. W.; Radke, C. J. A mechanistic population balance model for transient and steady-state foam flow in Boise sandstone. Chem. Eng. Sci. 1995, 50 (23), 3783-3799.

(15) Kam, S. I.; Nguyen, Q. P.; Li, Q.; Rossen, W. R. Dynamic simulations with an improved model for foam generation. SPE J. (Soc. Pet. Eng.) 2007, 12 (1), 35-48.

(16) Chen, Q.; Gerritsen, M. G.; Kovscek, A. R. Modeling foam displacement with the local equilibrium approximation: Theory and experimental verification. SPE J. (Soc. Pet. Eng.) 2010, 15 (1), 171183.

(17) Patzek, T. W.; Myhill, N. A. Simulation of the Bishop Steam Foam Pilot. In SPE California Regional Meeting (SPE 18786), Bakersfield, CA, 1989.

(18) Mohammadi, S. S.; Coombe, D. A.; Stevenson, V. M. Test of steam-foam process for mobility control in South-Casper Creek reservoir. J. Can. Pet. Technol. 1993, 32 (10), 49-54.

(19) Cheng, L.; Reme, A. B.; Shan, D.; Coombe, D. A.; Rossen, W. R. Simulating foam processes at high and low foam qualities. In SPE/ DOE Improved Oil Recovery Symposium (SPE 59287), Tulsa, OK, 2000.

(20) Boeije, C. S.; Rossen, W. R. Fitting foam simulation model parameters to data. In 17th European Symposium on Improved Oil Recovery; St. Petersburg, Russia, 2013.

(21) Rossen, W. R.; Boeije, C. S. Fitting foam simulation model parameters for SAG foam applications. In SPE Enhanced Oil Recovery Conference (SPE 165282), Kuala Lumpur, Malaysia, 2013.

(22) Ma, K.; Lopez-Salinas, J. L.; Puerto, M. C.; Miller, C. A.; Biswal, S. L.; Hirasaki, G. J. Estimation of parameters for the simulation of foam flow through porous media. Part 1: The dry-out effect. Energy Fuels 2013, 27 (5), 2363-2375.

(23) Aronson, A. S.; Bergeron, V.; Fagan, M. E.; Radke, C. J. The influence of disjoining pressure on foam stability and flow in porous media. Colloids Surf., A. 1992, 83 (2), 109-120.

(24) Exerowa, D.; Kruglyakov, P. M. Foam and Foam Films; Elsevier Science: Amsterdam, 1998

(25) Farajzadeh, R.; Krastev, R.; Zitha, P. L. J. Foam films stabilized with alpha olefin sulfonate (AOS). Colloids Surf., A 2008, 34 (1-3), $35-40$.

(26) Osterloh, W. T.; Jante, M. J., Jr. Effects of gas and liquid velocity on steady-state foam flow at high temperature. In SPE/DOE Enhanced Oil Recovery Symposium (SPE 24179), Tulsa, OK, 1992.

(27) Alvarez, J. M.; Rivas, H.; Rossen, W. R. A Unified Model for Steady-State Foam Behavior at High and Low Foam Qualities. SPE J. (Soc. Pet. Eng.) 2001, 6, 325-333.

(28) Computer Modelling Group Ltd. STARS User's Guide; Computer Modelling Group Ltd.: Calgary, Alberta, Canada, 2010.

(29) Coombe, D. A. Personal communication, 2012.

(30) Moradi-Araghi, A.; Johnston, E. L.; Zornes, D. R.; Harpole, K. J. Laboratory evaluation of surfactants for $\mathrm{CO}_{2}$-foam applications at the South Cowden unit. In SPE International Symposium on Oilfield Chemistry (SPE 37218), Houston, TX, 1997.

(31) Ransohoff, T. C.; Radke, C. J. Laminar flow of a wetting liquid along the corners of a predominantly gas-occupied noncircular pore. $J$. Colloid Interface Sci. 1988, 121 (2), 392-401.
(32) Bertin, H. J.; Quintard, M. Y.; Castanier, L. M. Modeling Transient Foam Flow in Porous Media Using a Bubble Population Correlation. SPE J. (Soc. Pet. Eng.) . 1998, 3 (4), 356-362.

(33) Kapetas, L.; Vincent-Bonnieu, S.; Farajzadeh, R.; Eftekhari, A. A.; Mohd Shafian, S. R.; Kamarul Bahrim, R. Z.; Rossen, W. R. Effect of permeability on foam-model parameters: An integrated approach from coreflood experiments through to foam diversion calculations. Paper presented at 18th European Symposium on Improved Oil Recovery, Dresden, Germany, April 14-16, 2014.

(34) Brooks, R. H.; Corey, A. T. Hydraulic Properties of Porous Media. Hydrology Paper, No. 3; Colorado State University: Fort Collins, CO, 1964.

(35) Brooks, R. H.; Corey, A. T. Properties of Porous Media Affecting Fluid Flow. J. Irrig. Drain. Div., Am. Soc. Civ. Eng. 1966, 92 (2), 61-90.

(36) Skjaeveland, S. M.; Siqveland, L. M.; Kjosavik, A.; Hammervold, W. L.; Virnovsky, G. A. Capillary Pressure Correlation for Mixed-Wet Reservoirs. In SPE India Oil and Gas Conference and Exhibition (SPE 39497), New Delhi, India, 1998.

(37) Li, K. Generalized Capillary Pressure and Relative Permeability Model Inferred From Fractal Characterization of Porous Media. In SPE Annual Technical Conference and Exhibition (SPE 89874), Houston, TX, 2004.

(38) Jorden, J. R.; Campbell, F. L. Well Logging 1: Rock Properties, Borehole Environment, Mud and Temperature Logging; SPE Monograph 9; Society of Petroleum Engineers of AIME (SPE): Dallas, TX, 1984.

(39) Rossen, W. R.; Zhou, Z. H. Modeling foam mobility at the limiting capillary pressure. SPE Advanced Technology Series 1995, 3, $146-152$.

(40) Rossen, W. R.; Lu, Q. Effect of capillary crossflow on foam improved oil recovery. In SPE Western Regional Meeting (SPE 38319), Long Beach, CA, 1997.

(41) Rossen, W. R.; Zeilinger, S. C.; Shi, J. X.; Lim, M. T. Simplified Mechanistic Simulation of Foam Processes in Porous Media. SPE J. (Soc. Pet. Eng.) 1999, 4 (3), 279-287.

(42) Jimenez, A. 1.; Radke, C. J. Dynamic Stability of Foam Lamellae Flowing Through a Periodically Constricted Tube. In ACS Symposium on Oil Field Chemistry, Toronto, Canada, 1988.

(43) Apaydin, O. G.; Kovscek, A. R. Surfactant Concentration and End Effects on Foam Flow in Porous Media. Transp. Porous Media 2001, 43 (3), 511-536.

(44) Burdine, N. T. Relative Permeability Calculations From Pore Size Distribution Data. J. Pet. Technol. 1953, 5 (3), 71-78. 